\title{
Ultrastructural Findings of the Endothelial Cells in Pancreatic Lesions after Chronic Administration of Methamphetamine in Rats
}

\author{
YOHKO ITO*, HIROFUMI JONO*,** AND HIDEKI SHOJO* \\ *Department of Forensic Medicine, Saga Medical School, Saga 849-8501 and \\ **Forensic Science Laboratory, Saga Prefectural Police Headquarters, \\ Saga 840-8540, Japan
}

\begin{abstract}
Summary: The effects of chronic administration of methamphetamine on the pancreas were studied using electron micrography in an experimental model. Methamphetamine $(1 \mathrm{mg} / \mathrm{kg} / \mathrm{day})$ was subcutaneously injected in five-week-old male Wistar Kyoto rats (WKY) for 8 weeks. Five age and sex-matched WKY served as controls. In light microscopy, scattered necrosis, intercellular vacuolization and severe hemorrhage were the prominent lesions in the methamphetamine-treated rats. The associated ultrastructural alterations consisted of degenerated, swollen, mitochondria with disrupted cristae and cell debris in the superficial space of the endothelial cells, and extensive vacuolization in the degenerated endothelial cells of small vessels and in the smooth muscle cells of their wall. However, in this study, hypercontration or lamellar membrane-like changes were not identified. It is suggested that pancreatic necrotic hemorrhage is a consequence of methamphetamine damage to endothelial cells in various organs, including pancreas.
\end{abstract}

Key words methamphetamine, pancreas, ultrastructure, endothelial cells, mitochondria

\section{INTRODUCTION}

It is well known that autopsy cases of methamphetamine abusers show severe scattered hemorrhages in various organs including lungs, brain and pancreas. How methamphetamine causes death in these cases is still controversial. The pancreas is very vulnerable to hypovolemia, hypoxia, and some toxins or drugs. In rats, severe pancreatic lesions were observed at light microscopy after chronic administration of methamphetamine as reported previously [1].

In the present report, we describe the findings of an electron microscopic study of the pancreatic lesions in rats chronically treated with methamphetamine.

\section{MATERIALS AND METHODS}

Four-week-old male Wistar kyoto rats (WKY) were maintained for more than one week under a controlled environment before they were used in the study. They were fed commercial laboratory chow ad libitum throughout the experiment. Methamphetamine (Meth, Philopon, Dainippon Pharmaceutical) was dissolved in isotonic saline at a concentration of 1 $\mathrm{mg} / \mathrm{ml}$, and injected subcutaneously into five WKY at a dose of $1 \mathrm{ml} / \mathrm{kg}$ of body weight/day for 8 weeks. Body weights were recorded daily. Five age-matched male WKY received equal volumes of saline without methamphetamine in the same way. The rats were sacrificed under pentobarbital anesthesia and the pancreata were glutaraldehyde-fixed for histological study. The tissue samples were fixed with $10 \%$ neutral formalin, embedded in paraffin, cut into $4 \mu \mathrm{m}$ sections, and stained with hematoxylin-eosin or Masson's trichrome or Weigert's for elastic fibers. For electron microscopy, small tissue specimens were obtained from parts of the tail, head and body of the pancreas where various lesions including necrosis and hemorhages were definitely observed, 
as reported previously [2]. The tissue was further fixed in $2 \%$ glutaraldehyde-cacodylate, postfixed in $1 \%$ osmium tetroxide, dehydrated in graded alcohol, and embedded in epon 812. Semi-thin sections were stained with toluidine blue and examined with light microscopy. Ultra-thin sections were stained with both lead citrate and uranyl acetate and examined with JEM 100 CX type electron microscope. For ultrastructural investigation, pancreatic specimens were randomly taken from epon blocks.

\section{RESULTS}

With light microscopy, the lesions were similar to those reported previously [1]. Scattered edematous lesions and moderate vacuolization were demonstrated in the pancreas of 8 of the methamphetaminetreated rats. Severe regional hemorrhage, partial or total acinal cell necrosis, neutrophile infiltration, interstitial vessel dilatation, interstitial edema and fatty cell invasion were observed in 4 of the rats. In 2

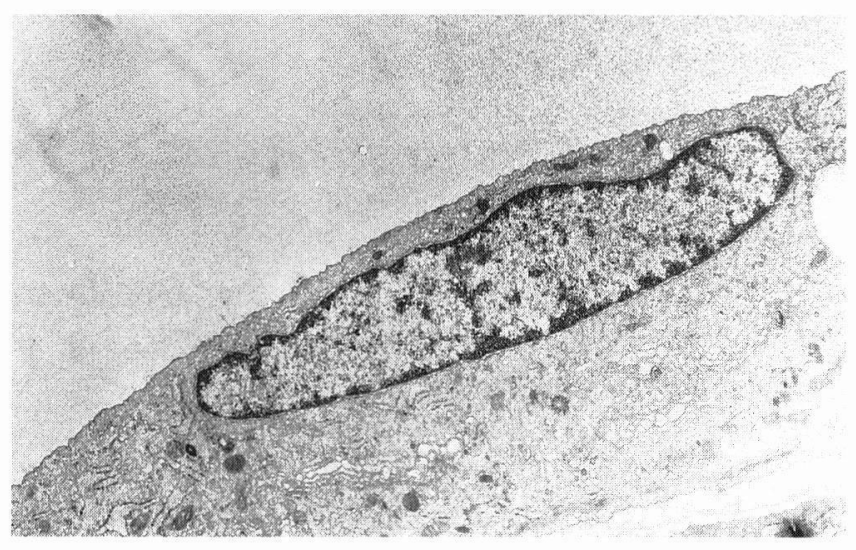

Fig. 1. Degenerated endothelial cell in the treated rat's pancreas. $(\times 12,000)$

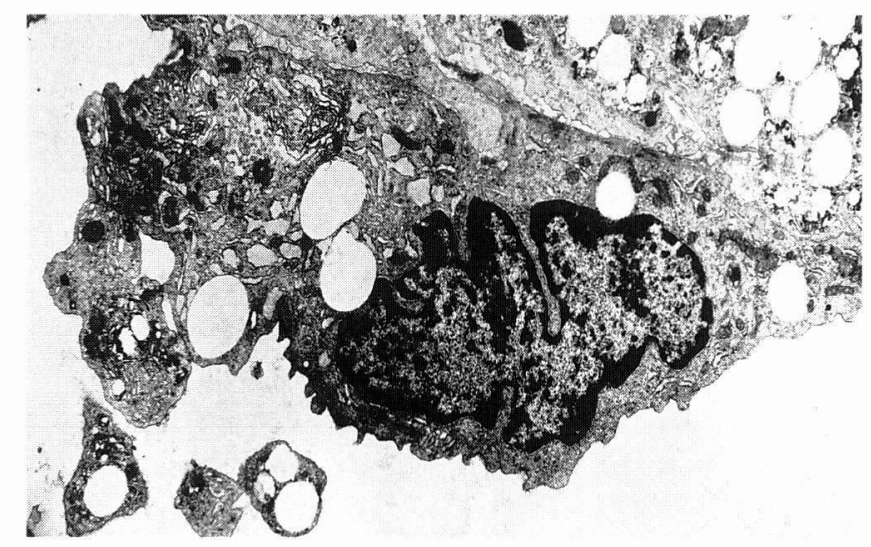

Fig. 2. Vacuolation and disruption of the cristae of mitochondria. $(\times 11,500)$ other animals, fibrosis and cirrhosis-like lesions with destruction and degeneration of the acinal cells were observed. Furthermore, the small blood vessels had moderate degeneration of the endothelial cells. In the control animals, no remarkable lesions were demonstrated. With electron microscopy, degenerated endothelial cells were shown in the Methamphetamine-treated rats near the hemorrhaging regions (Fig. 1). Degenerated endothelial cells were characterized by vacuolation and disruption of the cristae of mitochondria (Fig. 2). In the treated group, foci of disruption and lysis of myofibrils of the smooth muscles were frequently seen. Myofilaments were loosely arranged in myofibrils and the sarcoplasmic reticulum was dilated. Hypercontraction of sarcomeres was not shown. Vacuolization was moderate in the smooth muscle around the endothelial cells (Fig. 3). Intramyocytic vacuolization, seen in light microscopy, was not found in this study. Neither were lamellar membrane-like changes of degenerated mitochondria

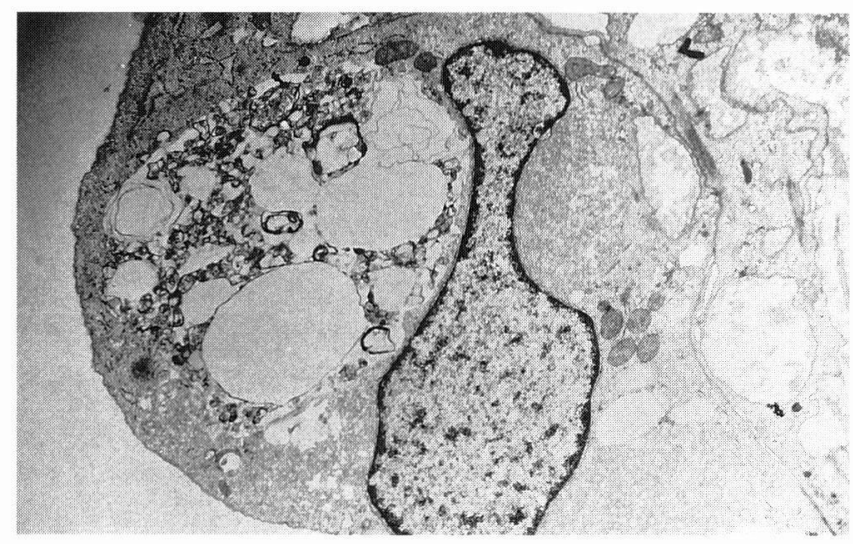

Fig. 3. Extensive vacuolization in the smooth muscle around the lesions. $(\times 12,000)$

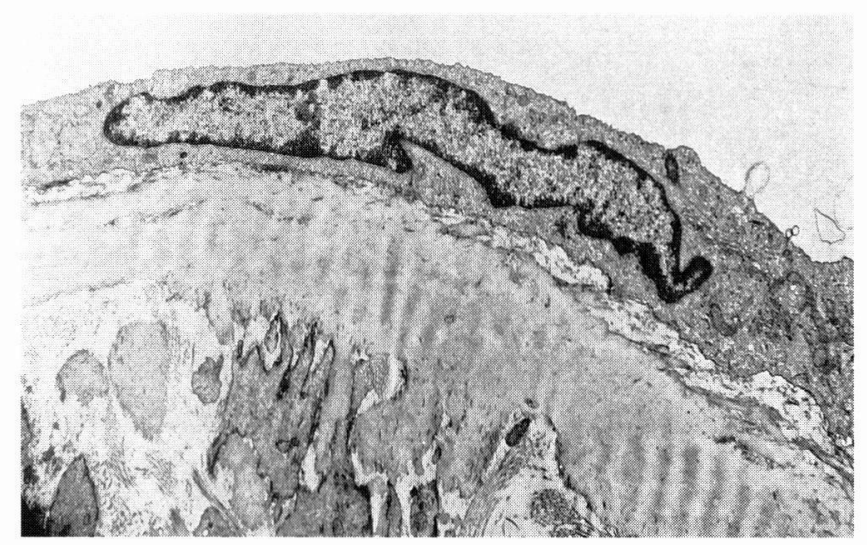

Fig. 4. Cell debris and supernatant plasma. $(\times 11,500)$ 
identified ultrastructurally. Only disruption of the cristae and swelling were found. Most mitochondria did not vary in size and shape, and did not aggregate abnormally in the perinuclear or submenbrane space. Severe disruption and fractions of mitochondria cristae were frequently found. Furthermore, many endothelial cells showed swelling just below the membrane. Cell debris and supernatant plasma were somewhere found in the superficial endothelial cells (Fig. 4).

\section{DISCUSSION}

The number of cases of sudden death among methamphetamine abusers has gradually increased in recents decades $[3,4]$. In some cases, after chronic administration of methamphetamine, victims suddenly die after complaining of an abnormal feeling in the heart, headache, nausea or severe abdominal pain. Of many organs which might be vulnerable to methamphetamine, we have studied the pancreas, because this organ is very vulnerable to hypoxia and toxins. In chronic methamphetamine-treated rats, an experimental model of human methamphetamine abuse, small but severe hemorrhages were demonstrated in the pancreas and also frequently in the lungs and the brain [1]. Recently, endothelial cell damage was shown to be related to toxic perfusion in various forms of toxic pancreatitis [2]. In the present study, we have examined changes in the methamphetamine-treated rat's pancreata. We show degenerative alterations and some mitochondrial changes in the endothelial cells of the small vessels near necrosis and hemorrhages. These changes were different from those we reported previously in myocardial cells of chronically methamphetaminetreated rats [5]. Furthermore, in other endothelial cells, some destruction mechanism might cause, for example, in the cases of arteriosclerosis, the alterations [6]. In the myocardial cells, light microscopy showed scattered cytomyolysis, vacuolization, contraction bands and disarray of myofibers in the methamphetamine-treated group; electron microscopy showed degenerated mitochondria with disrupted cristae, myofibrillar hypercontraction and dissolution. Morphometric analysis was carried out using electron photographs and an image analyzer. The size and the number of mitochondria per unit area (100 $\left.\mu \mathrm{m}^{2}\right)$ in the methamphetamine-treated group were significantly smaller than in the controls. No statistically significant difference was found regarding the percentage of myofibrillar area in the cytoplasm of myocardial cells between methamphetamine-treated and control groups. These findings show that chronic administration of methamphetamine can cause serious cardiac lesions and decrease in mitochondrial function. There have been several studies of the acute effects of methamphetamine. Zalis et al. [7] observed subendocardial hemorrhage, myocardial fiber necrosis and disruption of Purkinje fibers after administration of lethal doses of methamphetamine and amphetamine to unanesthetized dogs. Using immunohistochemical and ultrastructural methods, Kaiho and Ishiyama [8] found myocyte degeneration with myoglobin loss and swelling of mitochondria. The morphological features of the cardiac lesion were different between acute and chronic administration of methamphetamine in terms of their extent and degree. The mechanisms of cardiac lesions induced by methamphetamine are probably multifactorial. Recent in vitro studies have shown that methamphetamine is toxic to cardiomyocytes as assessed by LDH leakage and microscopic examination [9]. Biochemically, amphetamines inhibited selectively cell membrane-associated proteins and cytoskeleton proteins synthesis such as myosin, actin and tubulin in cultured chick myotubes [10]. It is well known that actions of methamphetamine on the heart are facilitation of catecholamine release and inhibition of presynaptic catecholamine uptake, which lead to an enhanced sympathetic state. The morphological alterations in the subendocardial and midventricular junctional areas might be a histological manifestation of chronic ischemia; vacuolar changes, atrophy of myocytes and fibrosis may be the results of hypoxia and chronic ischemia. The presence of scattered contraction bands, which develop as a reperfusion injury, supported the idea that the regions of the subendocardium and midventricular junction might be sensitive to ischemia. The mechanism for myocardial chronic ischemia and/or hypoxia still remains obscure. On the other hand, the cause of the pancreatitis due to some toxins has been controversial. Methamphetamine might have induced vasoactive changes, resulting in the degeneration of the small vessels of the pancreas or of the endothelial cells. Many studies in which pancreatitis was induced in rats and other experimental animals have demonstrated such lesions [11]. Calcium is a very important regulatory substance in pancreatic acinar cell function and a disturbance of calcium homeostasis could cause significant damage. Furthermore, indirect nonacinar events, such as ductal obstruction or ductal leakage, could be responsible for parenchym- 
atous damage. Konok et al. [12] stressed that the pancreatic ductal mucosa is a protective barrier against the pathogenesis of pancreatitis. Forceful injection of bile into the pancreatic duct, which presumably ruptures the ductal system, is followed by the development of acute pancreatitis. Shimosegawa et al. [13] histochemically investigated NADPH-diaphorase, a marker for NO synthase, in the rat pancreas. NADPH-diaphorase activity was localized to the neurons and the endothelium of the vessels. Nerve fibers with NADPH-diaphorase activity were observed in both the exocrine and the endocrine pancreas, associated chiefly with the vascular system. Furthermore in the evolution of acute pancreatitis, many workers using an ex vivo perfused canine pancreas model noted that ischemia from free fatty acid infusion leads to the development of pancreatic necrosis.

In view of these investigations, we will examine more thoroughly the Methamphetamine-treated rats and human autopsy cases to evaluate the practical legal autopsy of sudden death cases and try to determine the cause of the pancreatic apoplexy in chronic methamphetamine abusers. In addition to that, we think that it will be necessary to examine the endothelial cells of the brain's small vessels using electron microscopy.

ACKNOWLEDGMENTS: Part of this work was supported by the Grant-in-Aid (08670491) for Scientific Research from the Ministry of Education, Science and Culture, Japan.

\section{REFERENCES}

1. Ito $\mathrm{Y}$, Jono $\mathrm{H}$, and Shojo $\mathrm{H}$. A histopathological study of pancreatic lesions after chronic administration of methamphetamine to rats. Kurume Med J 1997; 44:209215.

2. Frick TW, Hailemariam S, Heitz PU, Largiader F, and
Goodale RL. Acute hypercalcemia induces acinar cell necrosis and intraductal protein precipitates in the pancreas of cats and guinea pigs. Gastroenterology 1990; 98:1675-1681.

3. Fujitani N, Matoba R, Shikata I, Tamata T, Mitsukuni Y et al. A statistical and histopathological study of autopsy cases of methamphetamine abusers for recent 5 years. Res Pract Forens Med 1990; 33:127-132.

4. Mizusawa I, Matsuo Y, Endo T, Aoki K, Kuroiwa Y et al. Statistical and histological studies on autopsy cases of methamphetamine abusers for recent 5 years. Res Pract Forens Med 1993; 36:283-290.

5. He SY, Matoba R, Sodesaki K, Fujitani N, and Ito Y. Morphological and morphometric investigation on cardiac lesions after chronic administration of methamphetamine in rats. Jpn J Legal Med 1996; 50:63-71.

6. Takebayashi S, and Kamio A. Ultrastructural studies on human arteriosclerosis. Comparison between hypertensive and normotensive groups. Acta Path Jap 1969; 19:479-499.

7. Zalis EG, Lundlberg GD, and Knutson RA. The pathophysiology of acute amphetamine poisoning with pathologic correlation. J Pharmacol Exp Ther 1967; 158: $115-127$

8. Kaiho H, and Ishiyama I. Morphological study of acute myocardial damage experimentally induced by methamphetamine. Jpn J Legal Med 1989; 43:460-468.

9. Welder AA. A primary culture system of postnatal rat heart cells for the study of cocaine and methamphetamine toxicity. Toxicol Lett 1992; 60:183-196.

10. Salomon P. The effect of amphetamine on cultured myotubes: selective inhibition of protein synthesis. Life Sci 1978; 23:1941-1950.

11. Smith HJ, Roche AHG, Jagusch MF, and Herdson PB. Cardiomyopathy associated with amphetamine administration. American Heart Journal 1976; 91:792-797.

12. Knock GP, and Thompson AG. Pancreatic ductal mucosa as a protective barrier in the pathogenesis of pancreatitis. The American Journal of Surgery 1969; 117:18-23.

13. Shimosegawa T, Abe T, Satoh A, Abe R, Kikuchi Y et al. Histochemical demonstration of NADPH-diaphorase activity, a marker for nitric oxide synthase, in neurons of the rat pancreas. Neuroscience Letters 1992; 148:67-70. 